\title{
Energy-Efficient Hybrid Hierarchical Routing scheme for overall Efficiency in WSN
}

\author{
Raghunandan G H ${ }^{\mathrm{a}}$, Dr. A ShobhaRani ${ }^{\mathrm{b}}$ \\ $a^{*}$ Department of Electronics and Telecommunication Engineering, BMS Institute of Technology and Management, Bangalore \\ India \\ ${ }^{b}$ Department of Electronics and Communication Engineering, BMS Institute of Technology and Management, Bangalore, India
}

Article History: Received: 10 November 2020; Revised 12 January 2021 Accepted: 27 January 2021; Published online: 5 April 2021

\begin{abstract}
Wireless Sensor Network are spatially distributed sensors intended to monitor different physiological conditions. Sensing and communicating data from one place to another consumes more energy, therefore the management of sensor energy is a very important factor. Energy utilization, synchronization, and a lifetime of the network are the main criteria in WSN. More energy is utilized by sensors that are distant from the base station. The gateway nodes are deployed to collect and relay information from nodes to the base station to resolve this problem. To decrease energy consumption, gateway nodes are deployed in the network. In this paper, a hybrid approach is used to increase the overall efficiency of the network in WSNs with time synchronization which increases the throughput of the network. The efficiency of proposed protocol based network has shown improvements in network lifetime, residual energy, data packets, and the throughput of the network. The performance of WSN of the proposed scheme is compared to other classical routing schemes and the proposed algorithm has proved its merit.
\end{abstract}

Keywords: Gateway Node, Wireless Sensor Network, Routing protocols, Base Station, Centrality, Cluster head

\section{Introduction}

Today due to an increase in the demand for continuous monitoring of physiological changes of different environments has led to major developments and growth of micro-sensors. Previously few industries use wired sensors; implementation provides deployment of sensor nodes more viable than before. Previously, there has been a study regarding applications of WSN such as monitoring of environmental changes, the agricultural field, military surveillance, and home automation. Deep research on routing protocols efficiency is being done considering the power constraints in WSNs .Recently there has been more scope in the field of WSNs and their applications because they are easy to deploy and are of low cost, have flexibility. Algorithms are used to solve the problem of power constraint without altering the standard. Local collaboration among sensors, suppression, data compression, redundant data, and avoidance of direct transmission too far distant sensors is of the major factors that influence algorithm designers to device unique distributed, scalable and energy-efficient solution for Wireless Sensor Networks. In common, the sensor nodes measure environmental conditions. The sensor node extracts some useful information by processing the raw sensor signals. The output of this processed signal is transmitted through direct communication or multi-hop communication with access points across other sensor nodes. In some situations, repeaters (RPs) are used for multi-hops, to support sensors installed outside the radio range. One of the components of the WSN is the base station which has more energy, computational, communication resources. Forwarding of data from wireless sensor network onto a server is done by the BS which acts as a gateway. has less energy consumption when compared to the communication subsystem. Figure 1 shows the arrangement of WSN. The energy required for transmitting one bit may consume as same as executing a few thousand instructions. Hence, communication must be traded for computation. In different environments, together with remote and hostile regions, where ad-hoc communications are a key element, WSNs are to be deployed in large numbers. For this reason, algorithms and protocols should concentrate on subsequent issues:

Lifetime enhancement: Sensor nodes should be energy efficient and the consumption of energy of the device should be less due to the limited energy resources. The radio power supply when not in use should shut off to conserve power of the node

- Fault tolerance and Robustness.

- Self-configuration. 


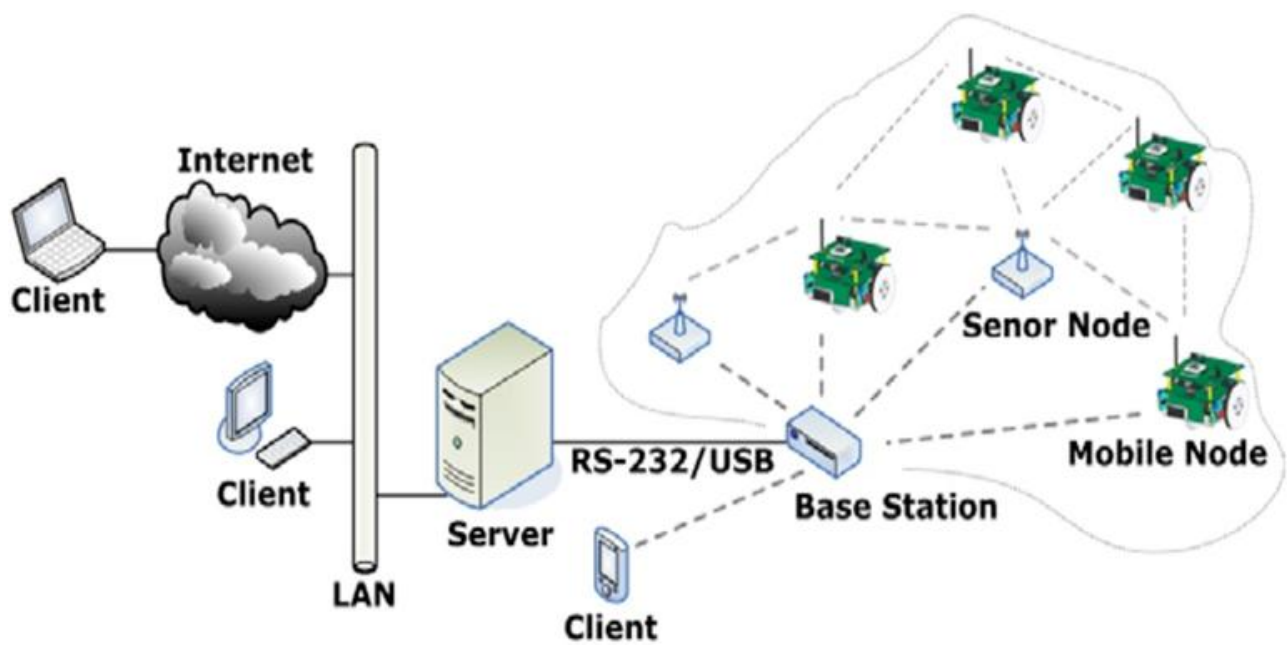

Figure 1: Schematic of WSN

Hierarchical routing algorithms are more efficient algorithms. In this, CHs are responsible for collecting the data and send it to GN and then the GN sends the information to the BS. If the members of the cluster are far, then there is wastage of energy. To solve this problem, the Centrality approach is used. In this method, the distance between every node to other nodes in the cluster is calculated using the Pythagoras theorem. The node which is equidistance to all other nodes along with high energy will be selected as the cluster head. This will reduce energy consumption. Error during communication can be reduced by using time synchronization in communication. This will reduce the packet collision during data transmission. In this paper, a new synchronization method considering a new broadcast sequence is used. This determines the order of the nodes that transmit timing information, this Eliminates collision. Section II presents a summary of the existing systems. The framework of the proposed system is explained in Section III. The system is evaluated in Section IV based on simulation and implementation. The paper concludes with section V.

\section{Existing System}

In [2] network is divided into areas as per geographic locations based on clustering for Cluster Head selection and formation in WSNs. $\mathrm{CH}$. After a few iterations, $\mathrm{CH}$ 's energy level decreases due to more energy usage as it processes the data obtained before forwarding it to the Base Station (BS). It is also no longer worthy of being the head of a cluster. Thus, the formation of the cluster takes place when $\mathrm{CH}$ residual energy is below the threshold goes; hence cluster reformation is not that efficient comparatively. In [3] It aims to minimize energy consumption at the level of the sensor node and the level of the network in the WSN. Before the available transmission, and then, the lowest transmission power required for transmitting, the distance between the transmitter and the receiver is calculated, but here the neighbor status information is less. In protocol [14] balance energy consumption is implemented for Cluster Heads (CHs) by creating clusters in each round with an almost equal number of nodes. But this is may not be applicable in all conditions as the nodes are randomly distributed based on applications. For transmission of data to the base station majority of the energy of sensor nodes is used. Thus, there is a fast depletion of energy. In [15] only energy conservation is concentrated which is the modified form of LEACH delay is not considered as a major factor.

Hierarchical Protocols opens up several technological challenges and enormous implementation possibilities as sensor networks interconnect many other nodes when large networks are set up. Using multi-hop wireless communications, these wireless sensor networks communicate. Due to the limited computational resources and finite power available to each sensor node, standard ad hoc routing methods cannot be directly applied to the domain of sensor networks. Protocols for Routing. In [6] for wireless sensor networks are used to ensure stable multi-hop communication and to maintain routes on the network. It provides us with an idea on routing protocols for Wireless Sensor Networks and compares their relative strengths and limitations to provide better energy efficiency or increase wireless sensor networks. Routing in WSNs is generally, classified into 3 types depending on the network structure. Data-centric routing protocols are often referred to as the first routing protocol. In this, each sensor node works together to perform the task of sensing. In the second kind of routing is hierarchical routing, higher energy nodes are used to process and send information, and low energy nodes are used to perform the sensing in the closeness of the target. In the third category location based routing, the sensor node's area is addressed through its location. By sharing such information between neighbors, relative co-ordinates of neighboring nodes can be obtained. In the current method, there are some routing problems and design concerns. One should assume the uniform distribution of sensor nodes when developing routing protocols; otherwise, we should go for clustering. Production costs should also be lower and service quality should be high. 


\section{Material Method}

In the recent past, wireless sensor network has attracted substantial research attention as WSN's are fastgrowing In hierarchical-based routing algorithms, Cluster Heads are in charge of compressing, forwarding and, gathering, data to the BS. Therefore, they play a major role. For the dissemination of all information to the members of that cluster. It is also critical that the clusters are developed for efficient WSN.CHs is also elected based on certain criteria. Thus efficiency, Network lifetime, and network stability of WSN are increased. In the Proposed algorithm, the sensor member senses the data and sends the data to the respective Cluster Heads. Cluster Head processes this data and sends it to the gateway node which in turn forwards to sink. Hence the efficiency of the $\mathrm{CH}$ increases which increases the network lifetime.

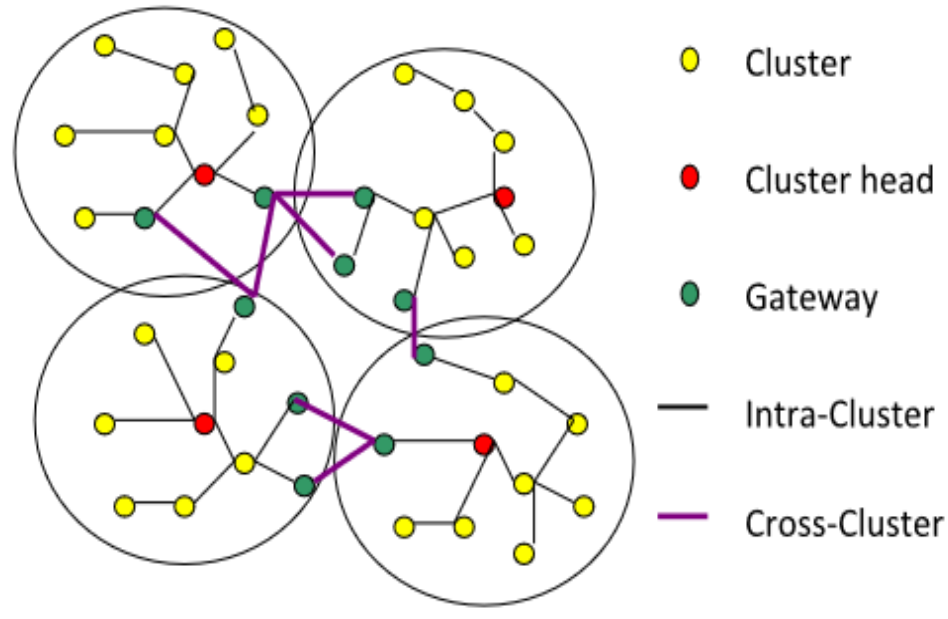

Figure 2: Clustering in WSN

Time synchronization is an elementary part of any network-oriented organization and system. In our proposed system grouping of sensor nodes is done called clustering. The transmitter's energy dissipated for the transmission of bit messages to a distance is provided by,

If the distance between the nodes is less than the threshold distance then the following equation will be used

Energy $=\left(\left(\mathrm{E}_{\mathrm{TX}}+\mathrm{E}_{\mathrm{DA}}\right)(\right.$ packet length $)+\mathrm{E}_{\mathrm{mp}}($ packet length $\left.)(\text { distance })^{2}\right)$

If the distance is greater than the threshold then the following equation will be used

Energy $=\left(\left(\mathrm{E}_{\mathrm{TX}}+\mathrm{E}_{\mathrm{DA}}\right)(\right.$ packetLength $)+\mathrm{E}_{\mathrm{fs}}($ packetLength $\left.)(\text { distance })^{4}\right)$

The energy dissipated by a receiver to receive bit message is given by

$\mathrm{E}_{\mathrm{R}}(\mathrm{k})=\mathrm{k}\left(\mathrm{E}_{\mathrm{RX}}\right)$

Threshold distance is given by

$r_{0}=\sqrt{\frac{E_{\mathrm{fs}}}{E_{\mathrm{mp}}}}$

Clustering is done to construct the appropriate topology of the network. The clustering-based network reduces the cost of the routing algorithm and the flooding broadcast. In this cluster-based routing approach, sensor nodes that are in a particular radio range are grouped which forms a cluster. Each group or cluster has one cluster head $(\mathrm{CH})$. This $\mathrm{CH}$ collects all the data from sensor nodes in a cluster and it performs data fusion and sends it to the nearby gateway node. $\mathrm{CH}$ is selected based on the centrality approach. In this method distance of nodes between each other is determined; the node which is equidistance from the other node and the node having more energy is selected as $\mathrm{CH}$. After $\mathrm{CH}$ selection aggregation of data is performed by removing redundant data. Data aggregation is the process of removing redundant data during transmission. This will increases the lifetime of the network. In our system base station (BS) is fixed. The same algorithm can also be applied to a movable base station. During the data communication phase, the data packets are transmitted from $\mathrm{CH}$ to the gateway node and from the gateway node to BS. This process is called multi-hop communication. The proposed technique uses the receiver-to-receiver concept implemented 
by the reference broadcast Synchronization which minimizes the time-critical path when compared to the sender-to-receiver method. The following assumptions are made for the proposed algorithm:

- The nodes have equivalent initial energy and are left unattained after deployment.But limitations on energy, memory, and computation are not considered for Base Station The nodes can limit the power required for transmission to the distance.

- Using wireless radio signal strength distance can be calculated.

- Usually, one of the causes of node failure is energy depletion.

- Sensor nodes are immobile.

Clustering is a process of connecting nodes using a specific topology to perform certain tasks as per the requirements. For wireless sensor networks, the algorithm used finds a collection of distinguished nodes to create the required network topology. The next step after the deployment of the sensor nodes is grouping the sensors into the cluster. In the proposed algorithm, cluster formation is the same as that employed in the LEACH algorithm. At the beginning of each round, after grouping nodes into clusters, the cluster head $(\mathrm{CH})$ is chosen. A threshold is set, up to which the proposed algorithm is used for the cluster head selection along with the centrality based approach. Data transmission is continuously monitored with energy updating after each round. The distance between one node and every other node is determined here, and the equidistant node is chosen as the head of the cluster. $\mathrm{CH}$ is selected based on certain criteria as mentioned below

1) $\mathrm{CH}$ 's residual energy: The $\mathrm{CH}$ is selected based on the maximum amount of energy it has.

2) The distance of the cluster head to the base station. The more distance from each other the more energy required for data transmission

3) Depends on the number of nodes in the cluster that is in a cluster if there are more members data processing required is also more.

In the Proposed Algorithm Agent gateway node approach is applied for the network. The Sensors are connected to corresponding $\mathrm{CH}$ and $\mathrm{CH}$ to nearby Gateway nodes. The Gateway nodes (GN) will be connected to the Base station (BS).Time Synchronization algorithm is applied between Sensors, CH, and GN. A reference node for time synchronization is selected for the first time. Later Source Receiver synchronization is applied and Beacon messages are sent to the nodes. Based on the radio energy dissipation model, the energy requirement of sensors are determined. The Routing of Data from nodes to $\mathrm{CH}, \mathrm{CH}$ to $\mathrm{GN}$, and the base station will be carried out.

\section{The Research Findings And Discussion}

The proposed system is simulated using MATLAB software. Here consider 100 nodes are distributed randomly along with gateway nodes. MATLAB Simulation is done considering the following parameters as shown in table 1 . The proposed algorithm results are compared with DR-LEACH , EPEGASIS , and ModLEACH. Figure 4 shows the initial topology of the hierarchical approach. Figure 4 shows LEACH topology. Figure 5 shows the topology of the proposed system. In the proposed system gateway node along with time synchronization is applied. Figure 6 illustrates the network's residual energy which is applied with the proposed algorithm. Figure 7 shows the presence of dead sensors after around 1350 rounds. The number of dead nodes of other classical routing schemes is more compared to the proposed system.

Table 1: Network Considerations

\begin{tabular}{|l|l|}
\hline Parameter & Considerations \\
\hline Initial Energy of sensor & $10 \mathrm{~J}$ \\
\hline Energy consumed to transmit one bit & $50 \mathrm{~nJ}$ \\
\hline Number of bits per packet & $6400 \mathrm{bits}$ per packet \\
\hline Energy for $\mathrm{E}_{\mathrm{fs}}$ & $10 \mathrm{pJ} / \mathrm{bit} / \mathrm{sq} \cdot \mathrm{m}$ \\
\hline Energy for $\mathrm{E}_{\mathrm{mp}}$ & $5 \mathrm{~nJ} / \mathrm{bit} / \mathrm{signal}$ \\
\hline Number of Sensor Nodes & 100 \\
\hline
\end{tabular}




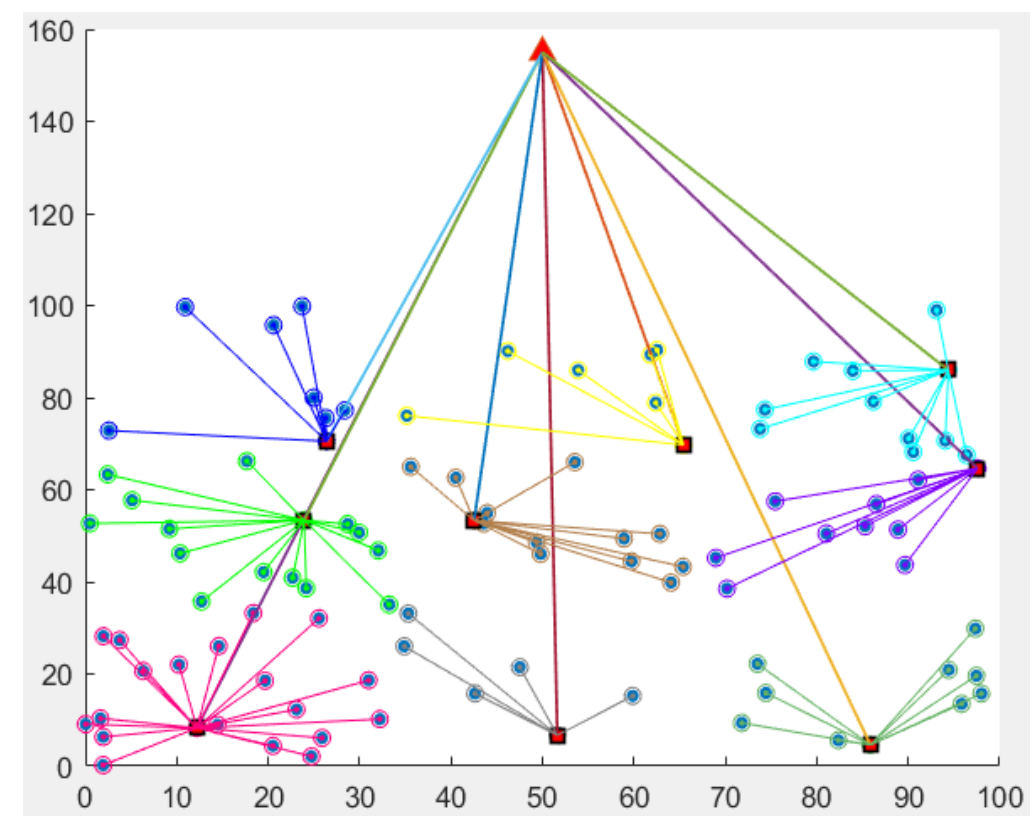

Figure 4: Initial Topology of LEACH

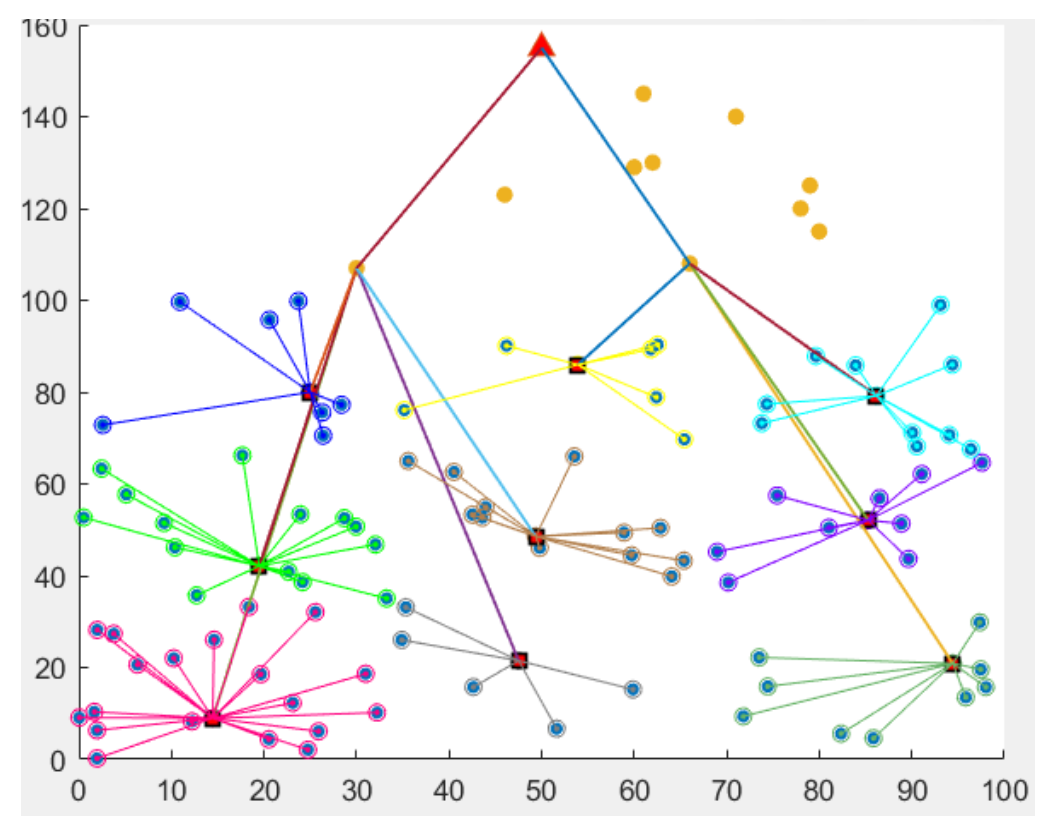

Figure 5: Proposed System

Figure 8 shows the number of packets transmitted to the base station to rounds. Here also the improvement in the number of data packets to the base station is more in the proposed algorithm compared to other routing schemes. The is a considerable lifetime enhancement in the proposed system compared to other schemes as shown in Figure 9.Figure 10 shows the delay in transmitting the data to the base station the proposed system shows less delay compared to other routing schemes. The delay is reducing gradually as synchronization among the nodes improved. The throughput of the network using different routing schemes is shown in Figure 11.here also the proposed algorithm shows a considerable improvement. This illustrates that the proposed system is more efficient compared to the existing protocols which are considered for Comparision. Throughput is calculated using the formula given below,

Throughput $=[$ Sum (number of successful packets)*(average packet length)]/ (Time taken by each round) 


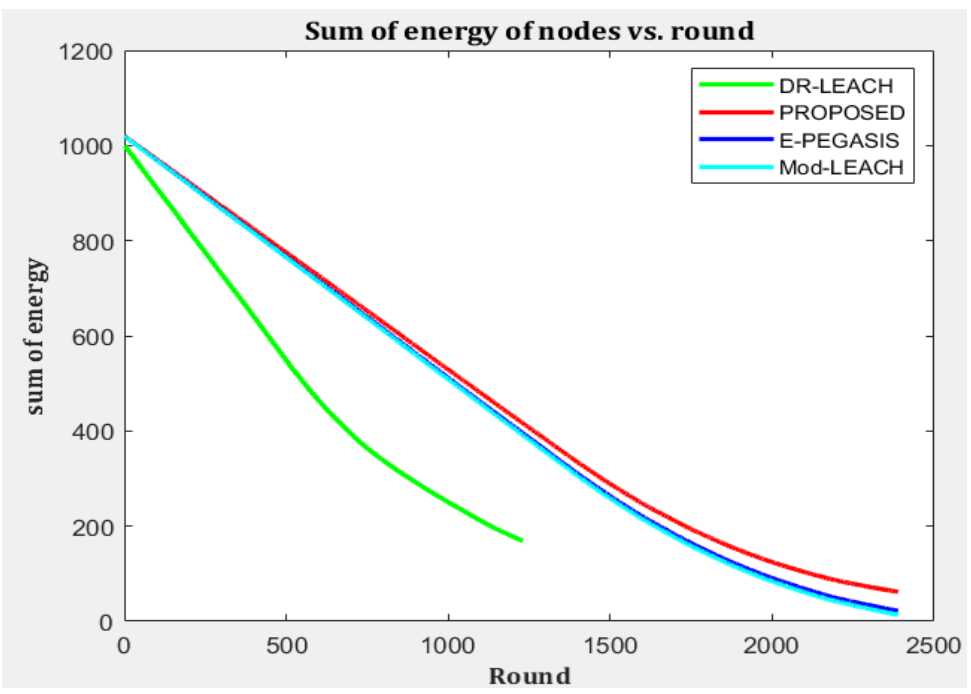

Figure 6: Comparision of Residual Energy of proposed system DR-LEACH, EPEGASIS, and Mod-LEACH

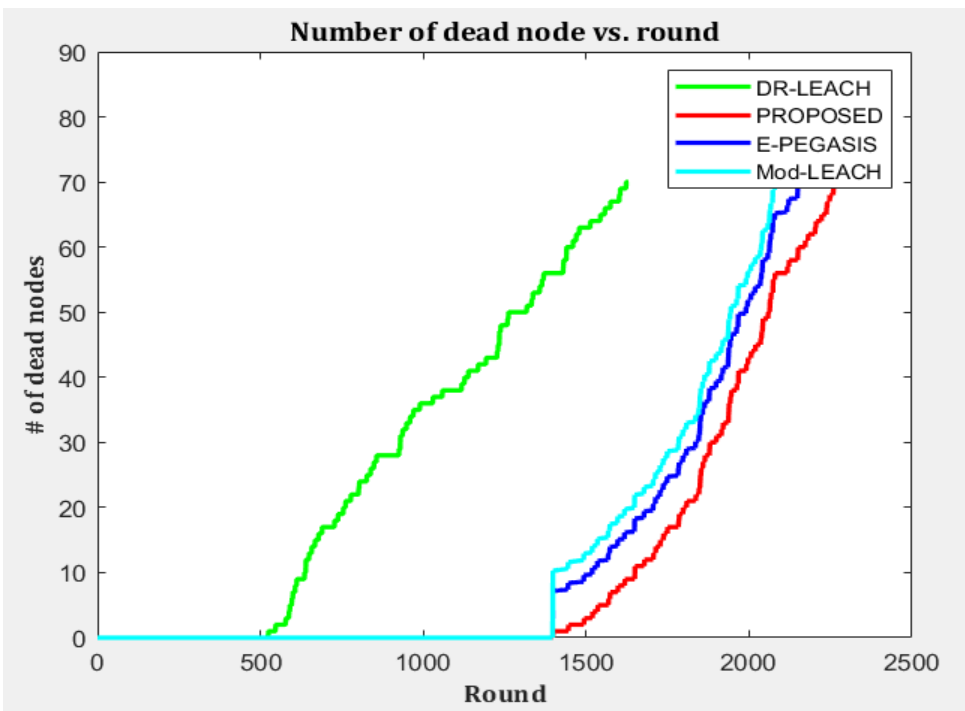

Figure 7:Comparision of number of dead nodes of the proposed system with DR-LEACH, EPEGASIS, and Mod-LEACH

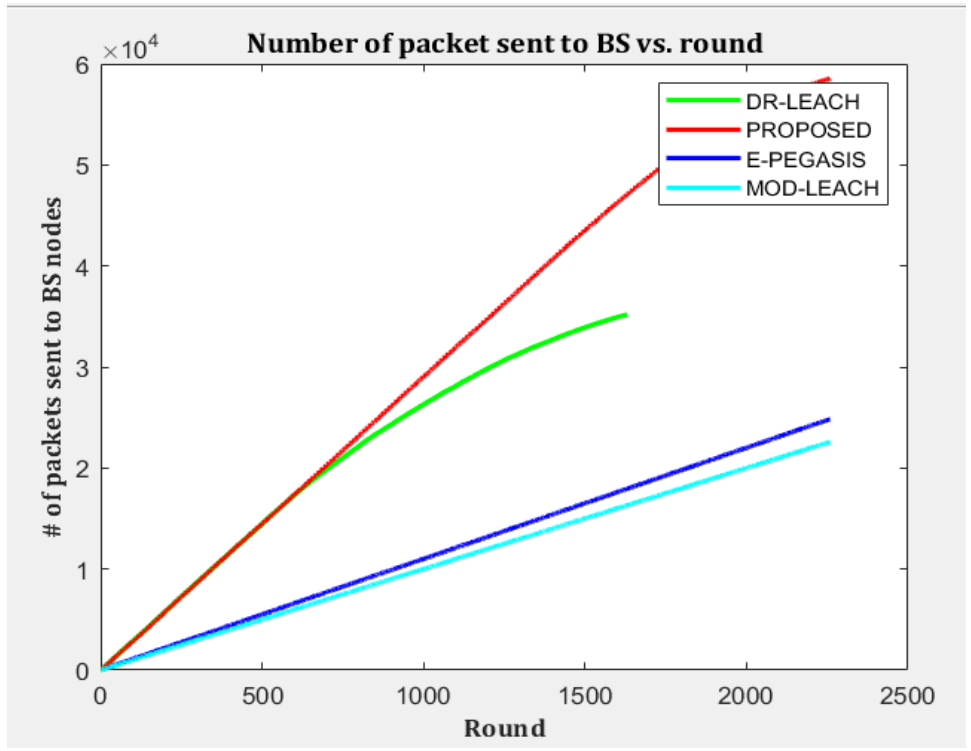

Figure 8: Comparision of packet delivered by the proposed system with DR-LEACH, EPEGASIS, and ModLEACH 


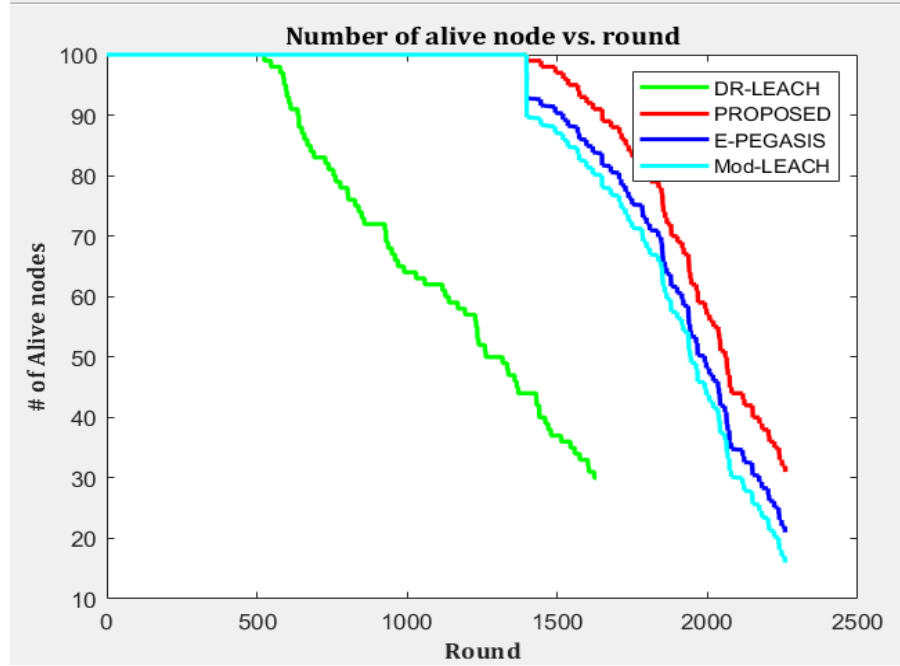

Figure 9:Comparision of Network Lifetime of Proposed system with DR-LEACH, EPEGASIS, and ModLEACH

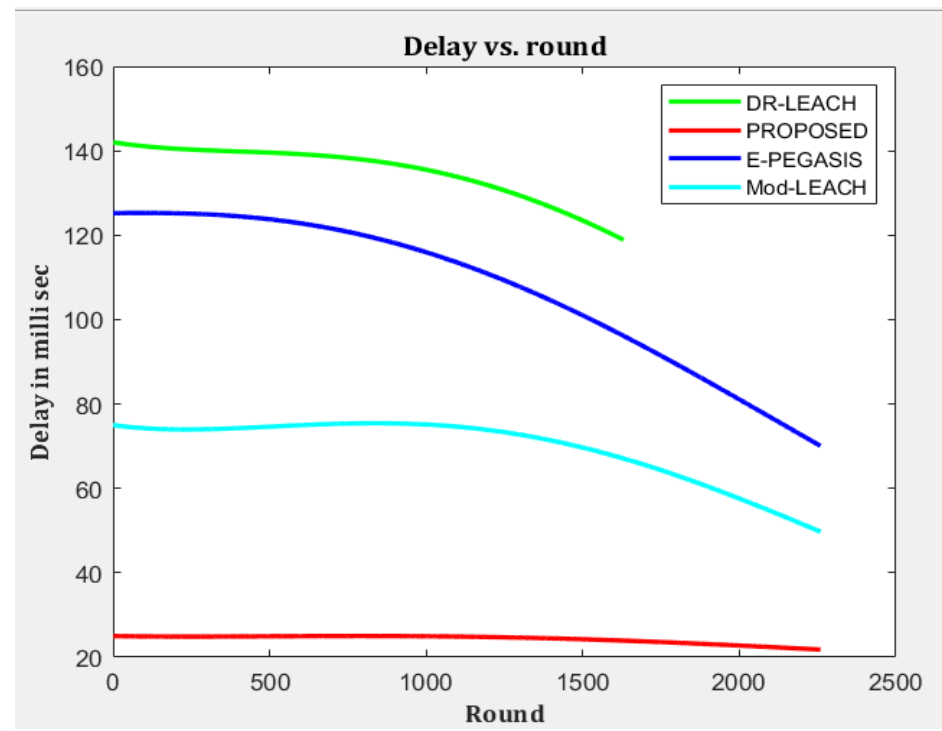

Figure 10: Comparision of delay by the proposed system with DR-LEACH, EPEGASIS, and Mod-LEACH

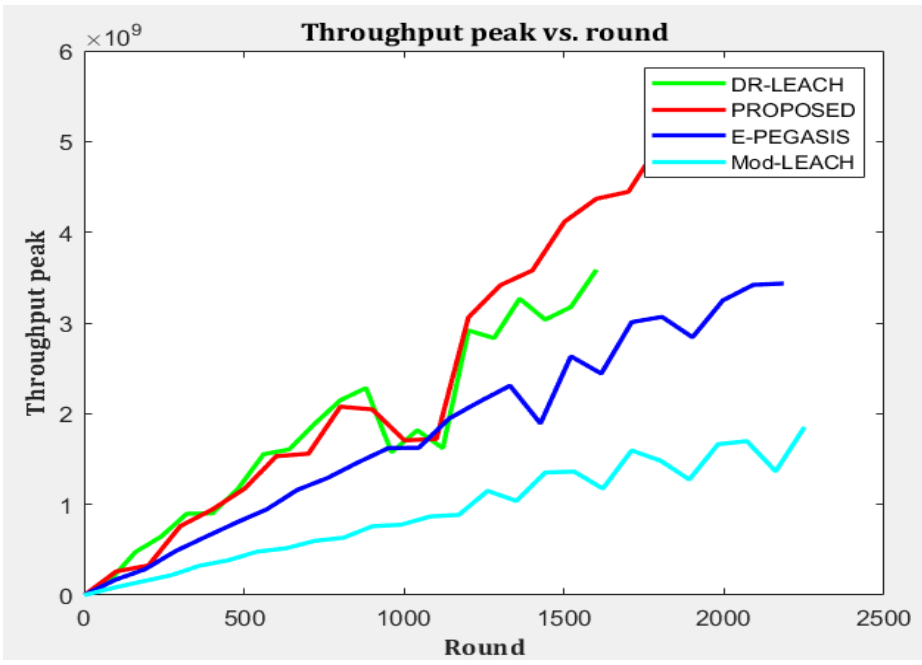

Figure 11: Comparision of Throughput by the proposed system with DR-LEACH, EPEGASIS, and ModLEACH 
Here we are comparing our proposed system with leach protocol. Figure 9 indicates the presence of alive nodes after corresponding iterations. The number of dead sensors appears nearly around 1000 iteration as shown in Figure 10. Hence the network lifetime is improved and the residual energy after each round provides us the information about the amount of energy consumed as in figure 11. Hence there is an improvement in the lifetime of the network of our proposed system.

\section{Results}

Energy consumption by sensors is a major parameter for network lifetime in WSN. In hierarchical clustering methodology, the cluster head selection plays an important role. Power consumption is one of the main factors for network lifetime in the wireless sensor network. By alternating this cluster head within the individual clusters, power consumption can be optimized by considering whether the network is taken as a whole. The choice of the cluster head between the nodes of the wireless sensor is based on the method of centrality. The implementation of gateway nodes would also reduce the load on the cluster head, which in turn extends the lifetime of the network. The outcome of the proposed approach is an improvement in the network lifespan and the number of nodes that remain alive for the maximum period. An efficient technique that is required for reducing the energy consumption by the nodes in the wireless sensor network is discussed. Along with that time synchronization is achieved using reference broadcast synchronization. The implemented protocol shows an improvement in the lifetime network, more data packets are sent with less delay. The throughput of the network using the proposed system is more compared to classical routing schemes. Here the proposed system is compared with the DR-LEACH, EPEGASIS, and Mod-LEACH protocol and it proves that the proposed system is more efficient than DR-LEACH, EPEGASIS, and Mod-LEACH.

\section{Conflicts Of Interest}

No conflict of interest was declared by the authors.

\section{References}

Salah M., Boulouz, "Energy Efficient Clustering based on LEACH", International Conference on Engineering and MIS, (2016).

D Djenouri, "R4 Time Synchronization in WSN", IEEE signal processing letters, Vol. 19, issue 4, (2012).

Sang H. K, Nguyen, "Distance-Based Thresholds for CH selection in WSN", IEEE Communications Letters, Vol 16, Issue 9, (2012).

Raghunandan, Sagar, "A Novel Approach to increase overall efficiency in WSN ",2012 IEEE ICCEET proceedings, pp 699-703, March 2012.

Raghunandan, Lakshmi, "A Comparative Analysis of Routing Techniques for WSN", IEEE NCOIET-2011, IEEE Conference Proceedings, (2011).

Akkaya, Younis, "A Survey of Routing Protocols in WSN" Journal of Ad Hoc Network, Elsevier, vol. 3, pp. 325-349, (2005).

Ying H, Chin Y, Ting C, " AER Protocol for mobile sink in WSN," IEEE Conference on Media Computing, conference proceeding, pp. 44-49, (2008).

Raghunandan.G H, Lakshmi "Secure Routing protocols for WSN", International Conference on FCS proceedings, (2011).

Yunhe, Zhihua, Zhang Q, "Efficient Load Balance Data Aggregation methods for WSN based on compressive network coding”, IEEE International Conference on EICT, (2016).

Amit R, R Singh, A Nandini “WSN - Challenges and Possibilities”, IJCA, volume 140, no 2, (2016).

Moumita, Paramita C, Sarmistha, Chowdhury "Analysis of EE WSN Routing Schemes", International Conference of EAIT, (2014).

Abhishek C, Sumedha, "Minimization of Avg Energy Consumption to Prolong Lifetime of WSN", IEEE Global Conference on WCN, (2014).

Zhenpengp,wuxiongzhang et.al," Energy-efficient improvements in mod-LEACH protocol on optimal cluster head selection", IEEE conference on IoT, Green computing and communication, (2018).

JinWang, Yu Gao, Xiang Y, Feng,"An Enhanced PEGASIS Algorithm with Mobile Sink Support for WSNs" Hindawi, Wireless Communications and Mobile Computing, Volume 9,(2018).

Abdallah I, Abdalraheem I, Huthaifa Al-Issa," Dynamic Re-Clustering LEACH-Based Protocol For WSNs", IJCNC, Vol.7, No.6, (2015). 